\title{
Saccharomyces cerevisiae-like 1 (SEC14L1) is a prognostic factor in breast cancer associated with lymphovascular invasion
}

\author{
Sultan N Sonbul ${ }^{1,2} \cdot$ Mohammed A Aleskandarany ${ }^{1,3} \cdot$ Sasagu Kurozumi ${ }^{1} \cdot$ Chitra Joseph $^{1} \cdot$ Michael S Toss $\mathbb{1}^{1}{ }^{1}$. \\ Maria Diez-Rodriguez ${ }^{1}$. Christopher C Nolan ${ }^{1} \cdot$ Abhik Mukherjee $^{1} \cdot$ Stewart Martin $^{1} \cdot$ Carlos Caldas $^{4} \cdot$ Ian 0 Ellis $^{1}$. \\ Andrew R Green ${ }^{1} \cdot$ Emad A Rakha ${ }^{1,3}$
}

Received: 26 March 2018 / Revised: 29 May 2018 / Accepted: 29 May 2018 / Published online: 28 June 2018

(c) United States \& Canadian Academy of Pathology 2018

\begin{abstract}
Lymphovascular invasion is strongly related to breast cancer metastasis. However, the underlying mechanisms of lymphovascular invasion and its driver molecules in breast cancer remain to be defined. In this study, we explore differential expression of genes in large molecularly characterized and clinically annotated datasets of invasive breast cancer patients $(n$ $=8056$ ) coupled with histological review and strict definition for lymphovascular invasion status. The METABRIC series was used to identify genes associated with lymphovascular invasion, as defined using hematoxylin and eosin staining supplemented by immunohistochemistry, at the genomic/transcriptomic levels. Saccharomyces cerevisiae-like 1 (SEC14L1) was identified as one of the most significant genes associated with lymphovascular invasion. The prognostic significance of SEC14L1 gene copy number and mRNA expression was further investigated in the METABRIC series and externally validated using the Breast Cancer Gene-Expression Miner v4.0. Protein expression of SEC14L1 was also assessed using immunohistochemistry in series of early stage breast cancer using tissue microarrays. SEC14L1 gene copy number gain was significantly associated with high histological grade and poor outcome. SEC14L1 mRNA expression showed positive association with higher grade, lymph node metastasis, and poor outcome. SEC14L1 protein overexpression was significantly associated with lymphovascular invasion $(p<0.0001)$, higher grade $(p=0.011)$, HER2 positivity $(p=0.036)$, and shorter survival $(p=0.00075)$. Our findings specify SEC14L1 as an independent prognostic factor in breast cancer. Its association, at both transcriptome and protein expression levels, with lymphovascular invasion and outcome could imply an important role in tumor progression. A further mechanistic insight into its molecular roles including potential therapeutic utility is warranted.
\end{abstract}

Electronic supplementary material The online version of this article (https://doi.org/10.1038/s41379-018-0092-9) contains supplementary material, which is available to authorized users.

Emad A Rakha

Emad.Rakha@nottingham.ac.uk

1 Division of Cancer and Stem Cells, School of Medicine, University of Nottingham and Nottingham University Hospital NHS Trust, Nottingham City Hospital, Nottingham, UK

2 Faculty of Sciences, Biochemistry Department, King Abdulaziz University, Jeddah, Saudi Arabia

3 Faculty of Medicine, Menofia University, Shibin Al Kawm, Egypt

4 Addenbrooke's Hospital, Cambridge Breast Unit, Cambridge University Hospital NHS Foundation Trust, Cambridge, UK

\section{Introduction}

Although several biomarkers associated with breast cancer progression and response to therapy have been identified, the exact molecular signatures for invasion machineries that lead to metastatic disease remains to be defined. Deciphering the driver genes/proteins that dictate the biological metastatic behavior of breast cancer is essential to understanding cancer progressive mechanisms and opening novel avenues for therapeutic interventions. Lymphovascular invasion is an independent prognostic parameter of poor outcome in invasive breast cancer and is major prerequisite for the development of metastasis [1-3]. Comprehension of the molecular mechanisms underlying lymphovascular invasion in breast cancer and unveiling its key-players could lead to unique therapeutic targets and improve risk prediction [4-6]. However, the sophistication of the 
molecular mechanisms underlying lymphovascular invasion as part of the invasion-metastasis cascade with involvement of several genes, diverse signaling pathways and interactions of tumor microenvironment in addition to the subjectivity of lymphovascular invasion morphological assessment in clinical samples renders this task highly challenging [6].

Advancement in high-throughput molecular and bioinformatics techniques coupled with thorough pathological assessment could help decipher lymphovascular invasion molecular regulators in breast cancer. Therefore, we have interrogated transcriptomic profiles of the large-scale cohort of invasive breast cancer patients of the Molecular Taxonomy of Breast Cancer International Consortium (METABRIC) following morphological and molecular assessment for lymphovascular invasion status to identify lymphovascular invasion differentially expressed genes [7]. Cytosolic factor Saccharomyces cerevisiae-like 1 (SEC14L1) was identified as one of the most significant genes associated with lymphovascular invasion using genomic data.

The aim of the present study was to evaluate the clinicopathological and prognostic significance of SEC14L1 copy number alteration and mRNA expression in the large annotated cohort of the METABRIC. Associations of SEC14L1 protein expression with clinicopathological prognostic variables including lymphovascular invasion, intrinsic molecular subtypes, and patient outcome were assessed. Our interpretation of the molecular and prognostic impact of SEC14L1 expression may unveil new insights on the complex pathways of lymphovascular invasion in breast cancer.

\section{Material and methods}

\section{Analysis of differential gene expression}

The METABRIC cohort [8, 9] is a large genomic and transcriptomic dataset from 1980 primary operable invasive breast cancer female patients. In this cohort, the estrogen receptor-positive and/or lymph node metastasis-negative patients were treated without adjuvant chemotherapy. The estrogen receptor-negative or lymph node-positive patients had adjuvant chemotherapy. No HER2-positive breast cancer patients had trastuzumab therapy. The extracted and purified DNA probes were hybridized to Affymetrix SNP 6.0 arrays (Affymetrix, Santa Clara, CA) at AROS Applied Biotechnology (Aarhus, Denmark). For RNA analysis, the Illumina Totalprep RNA amplification kit and Illumina Human HT-12 v3 Expression BeadChips (Ambion, Warrington, UK) were used as described previously [8].

We interrogated the Nottingham cases from the METABRIC cohort (discovery set) and gene expression levels were compared between lymphovascular invasionpositive and lymphovascular invasion-negative cases. The lymphovascular invasion status for all patients was evaluated morphologically using hematoxylin and eosin staining on full-face tumor sections. Besides, corresponding fullface tumor sections for each patient were stained immunohistochemically with CD34 and D2-40 (podoplanin, $P D P N)$, [4] to refine the status of lymphovascular invasion. Cases were considered lymphovascular invasion negative by the absence of lymphovascular invasion in both hematoxylin and eosin-, and immunohistochemistry-stained section. Cases with discordant results or showing lymph node positive but lymphovascular invasion negative were excluded, to avoid bias caused by false-positive or falsenegative diagnosis.

A linear model for microarray data and RNA-sequencing package (LIMMA) method [10] was applied for detecting differentially expressed genes/transcripts between lymphovascular invasion-positive and -negative groups. This supervised approach of differential gene expression analysis is compatible with the Affymetrix gene expression data. The Addenbrookes' Hospital cases, within the METABRIC cohort $(n=521)$, with defined lymphovascular invasion status were used to validate the results of the differential gene expression analyses (validation set). The top differentially expressed genes were ranked based on their $p$-value of association with lymphovascular invasion. Subsequently, the copy number alterations of the top differentially expressed genes were determined using the Affymetrix SNP6 Copy Number Inference Pipeline (Cancer Genomics Computation Analysis group of the Broad Institute, USA) [7]. Cytosolic factor SEC14L1 was identified as one of the highly significant genes associated with lymphovascular invasion. The prognostic impact of SEC14L1 mRNA expression was evaluated in the 1980 cases of METABRIC cohort and externally validated using the Breast Cancer Gene-Expression Miner v4.0 [ $n=5788]$ [11].

\section{Analysis of SEC14L1 protein expression}

The Nottingham breast cancer series, which were included in the METABRIC cohort, were used in this study. All patients were treated without neoadjuvant treatment. Estrogen receptor, progesterone receptor, HER2, and Ki67 were stained and scored according to guideline recommendations and as previously published $[12,13]$. The cutoff value of estrogen and progesterone receptors were determined as 1\% [14]. Estrogen receptor-positive and HER2negative tumors were considered as the luminal class with progesterone receptor-negative and high Ki67 (labelling index $<10 \%$ ) cases were determined as luminal B subtype. Clinicopathological data and patients' outcome had been recorded and regularly updated. The recorded outcome data 
Table 1 Association between SEC14L1 copy number alterations and SEC14L1 mRNA expression and clinicopathological parameters in the METABRIC cohort of invasive breast cancer $(n=1980)$

\begin{tabular}{|c|c|c|c|c|c|c|c|c|c|c|}
\hline \multirow[t]{2}{*}{ Factors } & & \multicolumn{5}{|c|}{ Expression of SEC14L1 (copy number alterations) } & \multicolumn{4}{|c|}{ Expression of SEC4L1 (mRNA) } \\
\hline & & Loss & Neutral & Gain & Total & $p$-Value & $>$ Median & $\leq$ Median & Total & $p$-Value \\
\hline \multirow[t]{2}{*}{ Tumor size } & $\geq 2 \mathrm{~cm}$ & $30(2.2 \%)$ & $1114(83.3 \%)$ & $193(14.4 \%)$ & 1337 & 0.063 & $657(49.1 \%)$ & $681(50.9 \%)$ & 1338 & 0.10 \\
\hline & $<2 \mathrm{~cm}$ & $13(2.1 \%)$ & $536(87.3 \%)$ & $65(10.6 \%)$ & 614 & & $327(53.1 \%)$ & $289(46.9 \%)$ & 616 & \\
\hline \multirow[t]{2}{*}{ Nodal status } & Positive & $20(2.1 \%)$ & $787(84.1 \%)$ & $129(13.8 \%)$ & 936 & 0.76 & $413(44.0 \%)$ & $525(56.0 \%)$ & 938 & $<0.0001$ \\
\hline & Negative & $23(2.2 \%)$ & $880(85.1 \%)$ & $131(12.7 \%)$ & 1034 & & $575(55.6 \%)$ & $460(44.4 \%)$ & 1035 & \\
\hline \multirow[t]{2}{*}{ Histological grade } & Grade 3 & $22(2.3 \%)$ & $764(80.3 \%)$ & $165(17.4 \%)$ & 951 & $<0.0001$ & $355(37.3 \%)$ & $597(62.7 \%)$ & 952 & $<0.0001$ \\
\hline & Grade 1,2 & $21(2.2 \%)$ & $834(88.9 \%)$ & $83(8.8 \%)$ & 938 & & $589(62.7 \%)$ & $351(37.3 \%)$ & 940 & \\
\hline \multirow[t]{5}{*}{ Molecular subtypes } & Luminal A & $14(2.0 \%)$ & $651(90.8 \%)$ & $52(7.3 \%)$ & 717 & $<0.0001$ & $481(67.0 \%)$ & $237(33.0 \%)$ & 718 & $<0.0001$ \\
\hline & Luminal B & $15(3.1 \%)$ & $358(73.4 \%)$ & $115(23.6 \%)$ & 488 & & $252(51.6 \%)$ & $236(48.4 \%)$ & 488 & \\
\hline & HER2 enriched & $5(2.1 \%)$ & $201(84.1 \%)$ & $33(13.8 \%)$ & 239 & & $78(32.5 \%)$ & $162(67.5 \%)$ & 240 & \\
\hline & Basal-like & $5(1.5 \%)$ & $275(83.8 \%)$ & $48(14.6 \%)$ & 328 & & $74(22.5 \%)$ & $255(77.5 \%)$ & 329 & \\
\hline & Normal-like & $4(2.0 \%)$ & $183(92.0 \%)$ & $12(6.0 \%)$ & 199 & & $101(50.8 \%)$ & $98(49.2 \%)$ & 199 & \\
\hline
\end{tabular}

comprised survival status, mean survival in months, recurrence of disease (including distant metastases), and cause of death. Breast cancer-specific survival was determined as the time from the day of surgery until the time of death from or with breast cancer.

Antigen-binding specificity for rabbit polyclonal antiSEC14L1 antibody (HPA028703, Sigma Aldrich, UK) was examined by western blotting (WB) at 1:500. A set of different cell lysates was utilized: MCF7, HeLa, MDA-MB231, Jurkat, human embryonic kidney 293, MDA-MB-468 (all obtained from the American Type Culture Collection, Rockville, MD, USA).

Before the immunohistochemistry assessment of SEC14L1 expression on tissue microarray, a subset of fullface tissue sections of breast cancer $(n=20)$ was immunohistochemically stained. Immunohistochemistry was performed on $(4 \mu \mathrm{m})$ tissue microarray sections using Novocastra Novolink Polymer Detection Systems kit (Code: RE7280-K, Leica Biosystems, UK) following the manufacturer's protocol. Briefly, all full-face and tissue microarray sections were incubated on a hot plate at $60^{\circ} \mathrm{C}$ for $10 \mathrm{~min}$, then deparaffinized in xylene and rehydrated through descending grades of ethanol. SEC14L1 staining was performed utilizing anti-SEC14L1 primary antibody at 1:25 dilution in Leica Antibody Diluent, for $60 \mathrm{~min}$. Finally, 3-3' Diaminobenzidine tetrahydrochloride (Novolink DAB substrate buffer plus) was freshly prepared and used as a chromogen. Counterstaining was performed using Meyer's hematoxylin for $6 \mathrm{~min}$. Positive (anti-human- $\beta$-2-microglobulin; A0072, Dako) and negative controls were included in the staining runs. The cytoplasmic expression of SEC14L1 was assessed using the percentage of positivity $(0-100 \%)$ and staining intensity (Negative: Score 0 , Weak: Score 1, Moderate: Score 2, and Strong: Score 3). H-score of SEC14L1 was calculated as previous publication [15].
This work was approved by Nottingham Research Ethics Committee 2 under the title: Development of molecular genetic classification of breast cancer. All tissue samples included in this study were from patients who were consented before inclusion in the study cohort.

\section{Statistical analysis}

Statistical analysis was performed using SPSS (IBM SPSS Statistics, Version 22). The relationship between SEC14L1 copy number alteration and mRNA expression was calculated using analysis of variance test with Bonferroni correction. Differences between two groups were assessed using Mann-Whitney test (non-normal distribution) to determine the associations between SEC14L1 mRNA expression and lymphovascular invasion status. $\chi^{2}$-test was used to evaluate the relationship between SEC14L1 expression and categorical variables. For dichotomization of the data, X-Tile (X-Tile Bioinformatics Software, Yale University, version 3.6.1) was used. Survival curves were generated by Kaplan-Meier survival analysis with differences in outcome assessed by Log Rank test. Cox's proportional hazard method was performed for multivariate analysis to identify the independent prognostic/predictive factors. The $p$-value $\leq 0.05$ was considered significant.

\section{Results}

There was a strong positive correlation between SEC14L1 mRNA expression and SEC14L1 gene copy number gain; higher levels of SEC14L1 mRNA were detected in cases with copy number gain compared with those with neutral copy number $(p<0.0001)$. SEC14L1 mRNA expression 

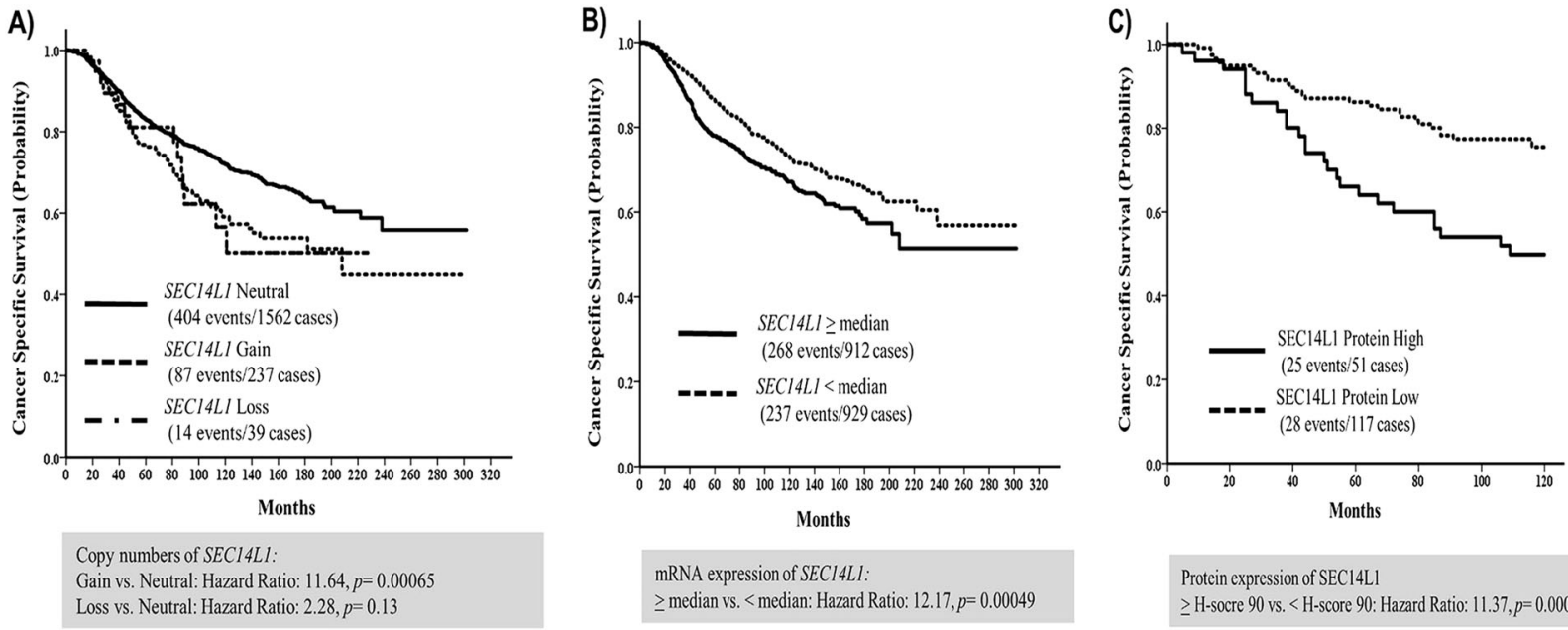

Fig. 1 Kaplan-Meier survival plots showing the association between SEC14L1 copy numbers (a), mRNA expression (b), and SEC14L1 protein expression (c) and outcome

was significantly lower in the copy number loss group $(p<$ 0.0001). Further analysis of SEC14L1 copy number alteration revealed a significant association with histological grade $3(p<0.0001)$ and luminal B molecular subtype $(p<$ 0.0001) (Table 1). The cutoff value of SEC14L1 mRNA expression was determined at median value. The overexpression of SEC14L1 mRNA was associated with the higher histological grade $(p<0.0001)$, axillary node metastasis $(p<0.0001)$, and the intrinsic molecular subtypes $(p<0.0001$; Table 1$)$.

In the METABRIC cohort, the survival of patients with SEC14L1 copy number gain was significantly shorter than those of copy number neutral group $(p=0.0007)$. However, no difference of survival was observed between copy number alteration loss and neutral groups $(p=0.13)$ (Fig. 1a). High expression of SEC14L1 mRNA conferred a significantly worse prognosis compared with low SEC14L1 mRNA expression ( $p=0.00049$; Fig. 1b). External validation of the prognostic power of SEC14L1 mRNA expression in the Breast Cancer Gene-Expression Miner v4.0 revealed that high SEC14L1 mRNA expression was associated with poor prognosis $(p<0.05)$.In the METABRIC cohort, the survival of patients with SEC14L1 copy number gain was significantly shorter than those of copy number neutral group $(p=0.0007)$. However, no difference of survival was observed between copy number alteration loss and neutral groups $(p=0.13)$ (Fig. 1a). High expression of SEC14L1 mRNA is relevant with a worse prognosis compared with low SEC14L1 mRNA expression ( $p=0.00049$; Fig. 1b). External validation of the prognostic power of SEC14L1 mRNA expression in the Breast Cancer GeneExpression Miner v4.0 revealed that high SEC14L1 mRNA expression was associated with adverse prognosis $(p<0.05)$.

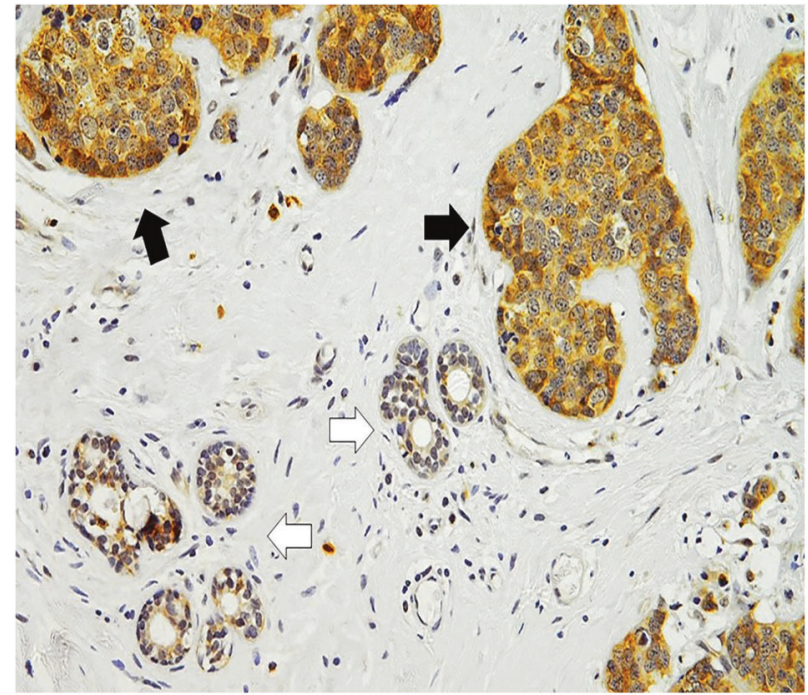

Fig. 2 SEC14L1 protein expression in normal and breast cancer tissue. Cytoplasmic SEC14L1 expression was overexpressed in breast cancer cells compared to the expression in epithelial cells of normal duct lobular units (black arrow: invasive carcinoma; white arrow: normal mammary gland)

\section{SEC14L1 protein expression}

Evaluation of WB supported a specific binding affinity of the anti-SEC14L1 primary antibody to single protein band for each cell lysate around the predicted molecular mass ( $77 \mathrm{kDa})$ of SEC14L1 protein, confirming the specificity of the antibody (Supplementary Figure 1). On full-face tissue sections, strong protein expression of SEC14L1 was detected in the cytoplasm of breast cancer cells (Fig. 2). On the other hand, weaker protein expression was observed in the adjacent normal epithelial tissue. Assessment of full-face sections revealed a homogeneous expression pattern for SEC14L1 protein 
A)

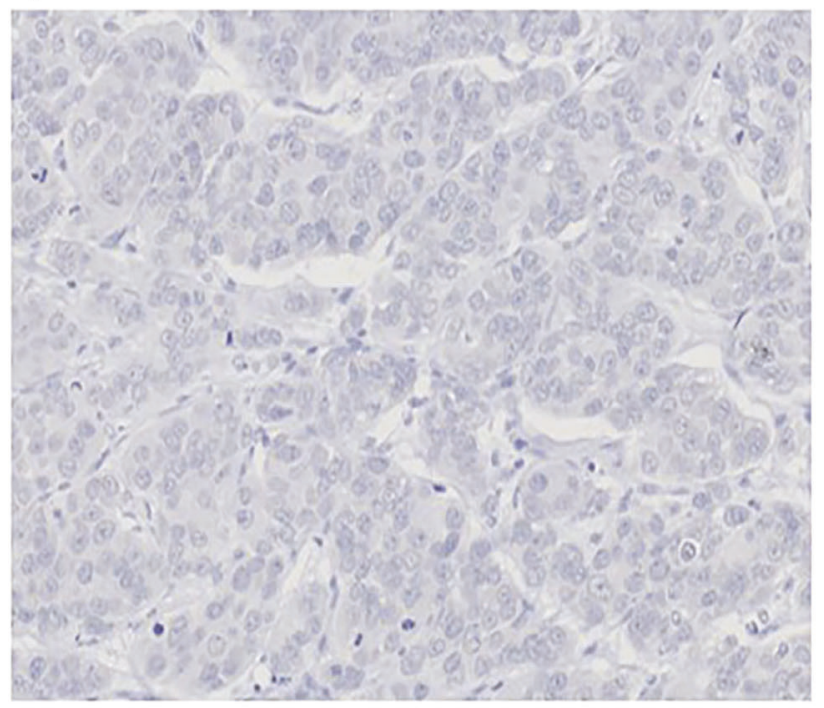

C)

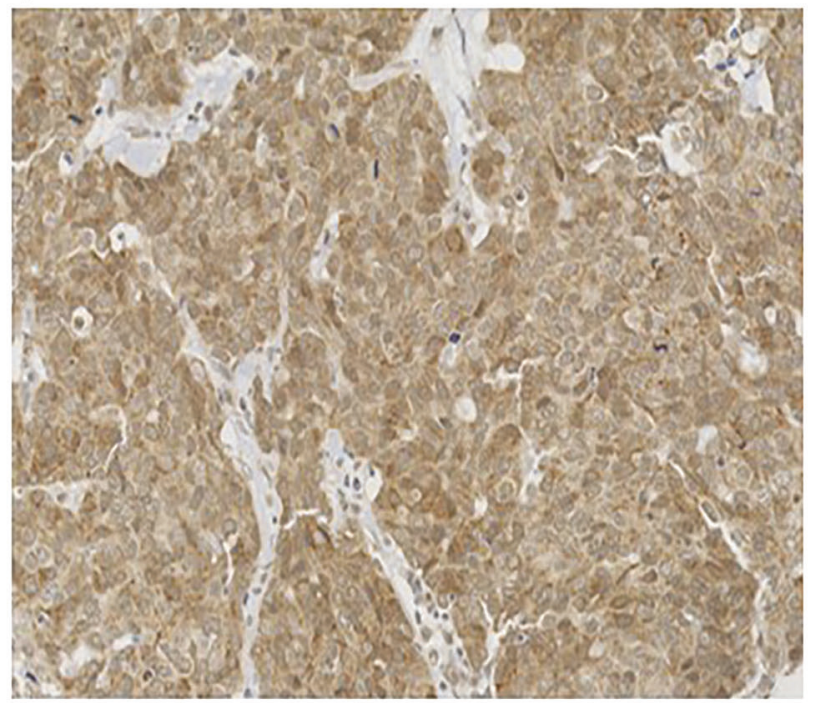

B)

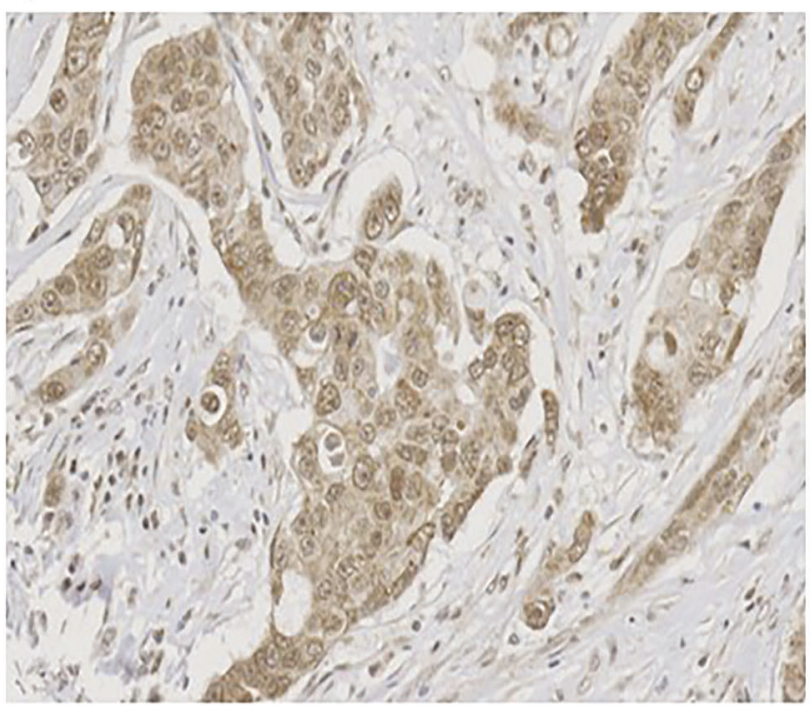

D)

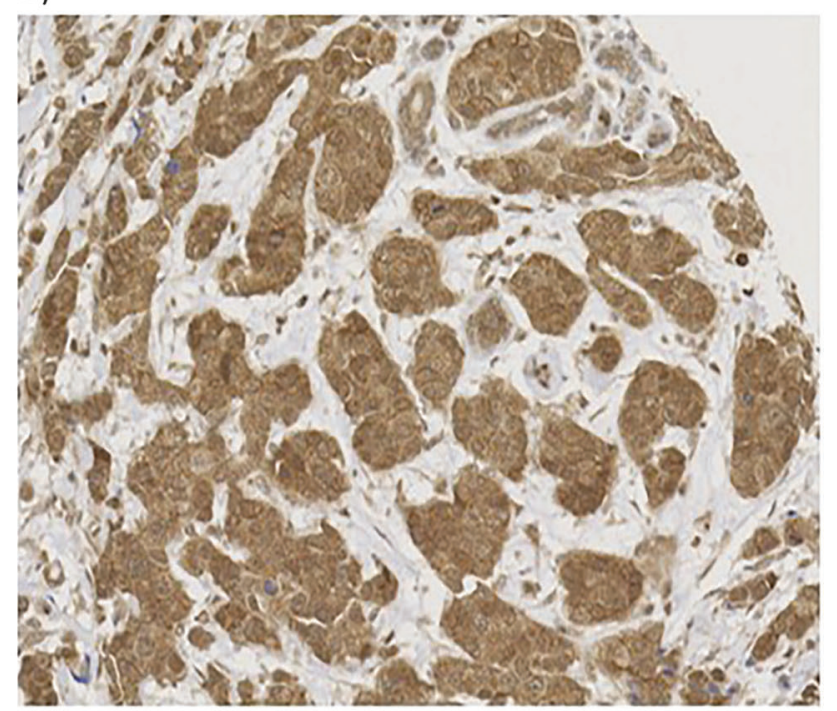

Fig. 3 Immunohistochemistry expression of SEC14L1 in tissue microarray images. SEC14L1 expression of the cytoplasm in cancer cells was distributed as follows; a no staining, b weak staining, c moderate staining, and $\mathbf{d}$ strong staining

confirming the reliability of tissue microarrays to detect its expression (Fig. 3). The optimal cutoff value of SEC14L1 protein expression was set at $\mathrm{H}$ score of 80 . At this cutoff, $70 \%$ cases showed low/negative cytoplasmic expression, whereas positive/high cytoplasmic expression was detected in $30 \%$.

There was an association between SEC14L1 protein expression and lymphovascular invasion status $(p<0.0001)$ and other variables of poor prognosis (Table 2). SEC14L1 expression was associated with a higher histological grade ( $p$ $=0.011)$, HER 2 positivity $(p=0.004)$, and luminal B subtype $(p=0.006)$. Outcome analysis revealed a positive association between SEC14L1 protein expression and shorter survival $(p=0.0008$; Fig. 1c). In univariate analysis,
SEC14L1 protein expression $(p=0.001)$, histological grade $(p<0.0001)$, lymphovascular invasion status $(p=0.002)$, tumor size $(p=0.005)$, and nodal status $(p=0.013)$ were all significant predictors of outcome. Using multivariate analysis including prognostic variables significant in univariate analysis, SEC14L1 protein expression was an independent prognostic variable of survival $(p=0.019$; Table 3$)$.

\section{Discussion}

In the present research, we have tried to decipher the molecular mechanism underlying lymphovascular invasion in breast cancer. High-throughput molecular techniques 
Table 2 Association between SEC14L1 protein expression and the clinicopathological factors

\begin{tabular}{|c|c|c|c|c|c|}
\hline \multirow[t]{2}{*}{ Factors } & & \multicolumn{4}{|c|}{ Expression of SEC14L1 } \\
\hline & & Low & High & Total & $p$-Value \\
\hline \multirow[t]{2}{*}{ Tumor size } & $\geq 2 \mathrm{~cm}$ & $68(68.7 \%)$ & $31(31.3 \%)$ & 99 & 0.75 \\
\hline & $<2 \mathrm{~cm}$ & $49(71.0 \%)$ & $20(29.0 \%)$ & 69 & \\
\hline \multirow[t]{2}{*}{ Nodal status } & Positive & $55(67.1 \%)$ & $27(32.9 \%)$ & 82 & 0.48 \\
\hline & Negative & $62(72.1 \%)$ & $24(27.9 \%)$ & 86 & \\
\hline \multirow[t]{2}{*}{ Histological grade } & Grade 3 & $53(60.9 \%)$ & $34(39.1 \%)$ & 87 & 0.011 \\
\hline & Grade 1,2 & $64(79.0 \%)$ & $17(21.0 \%)$ & 81 & \\
\hline \multirow[t]{2}{*}{ Lymphovascular invasion } & Positive & $43(51.8 \%)$ & $40(48.2 \%)$ & 83 & $<0.0001$ \\
\hline & Negative & $74(88.1 \%)$ & $10(11.9 \%)$ & 84 & \\
\hline \multirow[t]{2}{*}{ Estrogen receptor } & Positive & $85(69.1 \%)$ & $38(30.9 \%)$ & 123 & 0.80 \\
\hline & Negative & $32(71.1 \%)$ & $13(28.9 \%)$ & 45 & \\
\hline \multirow[t]{2}{*}{ Progesterone receptor } & Positive & $66(70.2 \%)$ & $28(29.8 \%)$ & 94 & 0.96 \\
\hline & Negative & $44(69.8 \%)$ & $19(30.2 \%)$ & 63 & \\
\hline \multirow[t]{2}{*}{ HER2 } & Positive & $14(53.8 \%)$ & $12(46.2 \%)$ & 26 & 0.036 \\
\hline & Negative & $98(74.2 \%)$ & $34(25.8 \%)$ & 132 & \\
\hline \multirow[t]{4}{*}{ Molecular subtypes } & Luminal A-like & $30(88.2 \%)$ & $4(11.8 \%)$ & 34 & 0.006 \\
\hline & Luminal B-like & $39(65.0 \%)$ & $21(35.0 \%)$ & 60 & \\
\hline & HER2-positive & $14(53.8 \%)$ & $12(46.2 \%)$ & 26 & \\
\hline & Triple negative & $23(85.2 \%)$ & $4(14.8 \%)$ & 27 & \\
\hline
\end{tabular}

Abbreviations: ER Estrogen receptor; $P g R$ Progesterone receptor coupled with bioinformatics and strict definition of lymphovascular invasion status were utilized to identify genes associated with lymphovascular invasion that can potentially be used as therapeutic targets. Following internal and external validation, SEC14L1 was identified as one of the top differentially expressed genes associated with lymphovascular invasion and also with prognosis. Further examination of SEC14L1 at protein expression level confirmed its association with lymphovascular invasion and indicated its independent prognostic value in breast cancer.

SEC14L1 gene is located within a discrete region of $17 \mathrm{q} 25$ that frequently shows copy number alterations in breast cancer [16]. A proximate locus, the $17 \mathrm{q} 23$ locus is well documented in breast cancer, and the key feature of this amplified locus is its oncogenic activity in breast cancer both in vitro and in vivo [17]. In this study, there was an association not only between SEC14L1 copy number alteration and mRNA expression but also between copy number alteration and mRNA expression and lymphovascular invasion status. In a recent investigation of prostatic carcinoma, a prognostic 12-gene signature, including SEC14L1, associated with criteria of aggressive clinical outcome, which was also confirmed by real-time quantitative and immunohistochemistry assays [18]. SEC14L1 cytoplasmic protein overexpression was frequently found in the Transmembrane Protease, Serine 2 (TMPRSS2)/ Etsrelated gene $(E R G)$ fusion-positive prostate cancer, and the clinical and prognostic power of SEC14L1 strongly depends on this fusion status in prostate cancer [19].
SEC14-like proteins have been implicated in hepatocellular carcinomas [20].

TMPRSS2/ERG fusion has been revealed to be associated with several molecular alterations including deletion of the phosphatase and tensin homolog gene (PTEN) [21]. Several previous studies suggested that PTEN loss is strongly correlated with transcriptional instability and was associated with poor outcome of HER2-positive breast cancer [22, 23]. TMPRSS2/ERG fusion was also thought to be correlated with androgen receptor activity [24], transcriptional stability [25], and stem cell maintenance [26] in cancer. These mechanisms have essential roles in cell proliferation, adhesion, invasion and migration. Thus, SEC14L1 may play a pivotal role in the regulation of cell growth/tissue development and cell adhesion, underlying lymphovascular invasion and metastasis in breast cancer.

Moreover, this study has revealed that SEC14L1 protein expression had a significant relationship with HER2 status and high histological grade. We previously reported that definite lymphovascular invasion was significantly correlated with HER2 positivity [1] and tumor microenvironment has a crucial role in the HER2 signaling pathway, invasion, and metastasis, including the development of lymphovascular invasion [27, 28]. A previous study suggested that SEC14L1 might have an important role in trafficking proteins on cell membrane [29]. SEC14L1 belongs to SEC14 cytosolic factor family that has a role in the intracellular transport system [30] and innate immunity [31]. SEC14L1 overexpression may be responsible for 
Table 3 Univariate and multivariate survival analysis of clinicopathological factors and SEC14L1 protein expression in breast cancer

\begin{tabular}{|c|c|c|c|c|c|c|c|}
\hline \multirow[t]{2}{*}{ Factors } & & \multicolumn{3}{|c|}{ Univariate analysis } & \multicolumn{3}{|c|}{ Multivariate analysis } \\
\hline & & $\begin{array}{l}\text { Hazard } \\
\text { ratio }\end{array}$ & $95 \% \mathrm{CI}$ & $p$-Value & $\begin{array}{l}\text { Hazard } \\
\text { ratio }\end{array}$ & $95 \% \mathrm{CI}$ & $p$-Value \\
\hline \multirow[t]{2}{*}{ SEC14L1 } & Low & Reference & & & Reference & & \\
\hline & High & 2.5 & $1.43-4.21$ & 0.001 & 2.0 & $1.12-3.54$ & 0.019 \\
\hline \multirow{2}{*}{$\begin{array}{l}\text { Lymphovascular } \\
\text { invasion }\end{array}$} & Negative & Reference & & & Reference & & \\
\hline & Positive & 2.5 & $1.41-4.47$ & 0.002 & 1.5 & $0.79-2.74$ & 0.22 \\
\hline \multirow[t]{2}{*}{ Tumor size } & $<2 \mathrm{~cm}$ & Reference & & & Reference & & \\
\hline & $\geq 2 \mathrm{~cm}$ & 2.4 & $1.30-4.43$ & 0.005 & 1.8 & $0.96-3.47$ & 0.067 \\
\hline \multirow[t]{2}{*}{ Nodal status } & Negative & Reference & & & Reference & & \\
\hline & Positive & 2.0 & $1.16-3.53$ & 0.013 & 1.4 & $0.76-2.47$ & 0.29 \\
\hline \multirow[t]{2}{*}{ Histological grade } & Grade $1-2$ & Reference & & & Reference & & \\
\hline & Grade 3 & 3.7 & $1.96-6.87$ & $<0.0001$ & 2.7 & $1.41-5.15$ & 0.003 \\
\hline
\end{tabular}

enhanced intra-tumor signaling pathways including HER2 and influence the tumor microenvironment to promote tumor growth, promoting lymphovascular invasion and metastasis in breast cancer. Although SEC14L1 was associated with higher histological grade, the association with outcome was independent of grade. In this study, SEC14L1 was associated with lymph node metastasis at mRNA levels, and its expression at mRNA and protein levels was associated with outcome independent of other prognostic variables.

In conclusion, this study revealed and confirmed that SEC14L1 expression is a significant prognostic factor for breast cancer. Overexpression of SEC14L1 may have an important role in the development of lymphovascular invasion, breast cancer progression, and metastasis. Further functional assessment of SEC14L1 to determine its mechanistic roles and therapeutic potential in breast cancer is warranted.

Acknowledgements We show our gratitude to Dr. Oscar Rueda (CRUK Cambridge Research Institute, University of Cambridge, Cambridge, UK) for providing the clinical, genomic, and transcriptomic profiles of the METABRIC patients in this study. We thank the Breast Cancer Research Trust and the University of Nottingham for funding this project, and the Nottingham Health Science Biobank (NHSB) and Breast Cancer Now Tissue Bank for the provision of tissue samples.

Author Contributions: REA, GAR, and EIO conceived of the study, contributed to study design, and provided samples and data. SSN, CC, MS, AMA, MA, TMS, and GAR collected data. D-RM and NCC provided technical support. SSN carried out experiments. SSN, AMA, JC, and KS analysed and interpreted data, and generated the figures and tables. All authors contributed to drafting and reviewing the manuscript, and approved the submitted and final version.

\section{Compliance with ethical standards}

Conflict of interest The authors declare that they have no conflict of interest.

\section{References}

1. Rakha EA, Martin S, Lee AH, et al. The prognostic significance of lymphovascular invasion in invasive breast carcinoma. Cancer. 2012;118:3670-3680.

2. Zhang S, Zhang D, Yi S, et al. The relationship of lymphatic vessel density, lymphovascular invasion, and lymph node metastasis in breast cancer: a systematic review and meta-analysis. Oncotarget. 2017;8:2863-2873.

3. Liu YL, Saraf A, Lee SM, et al. Lymphovascular invasion is an independent predictor of survival in breast cancer after neoadjuvant chemotherapy. Breast Cancer Res Treat. 2016;157:555-564.

4. Mohammed RA, Martin SG, Mahmmod AM, et al. Objective assessment of lymphatic and blood vascular invasion in lymph node-negative breast carcinoma: findings from a large case series with long-term follow-up. J Pathol. 2011;223:358-365.

5. Khwaja SS, Ivanovich J, DeWees TA, et al. Lymphovascular space invasion and lack of downstaging after neoadjuvant chemotherapy are strong predictors of adverse outcome in young women with locally advanced breast cancer. Cancer Med. 2016;5:230-238.

6. Aleskandarany MA, Sonbul SN, Mukherjee A, Rakha EA. Molecular mechanisms underlying lymphovascular invasion in invasive breast cancer. Pathobiology. 2015;82:113-123.

7. Aleskandarany MA, Sonbul S, Surridge R, et al. Rho-GTPase activating-protein 18: a biomarker associated with good prognosis in invasive breast cancer. Br J Cancer. 2017;117:1176-1184.

8. Curtis C, Shah SP, Chin SF, et al. The genomic and transcriptomic architecture of 2,000 breast tumours reveals novel subgroups. Nature. 2012;486:346-352.

9. Pereira B, Chin SF, Rueda OM, et al. The somatic mutation profiles of 2,433 breast cancers refines their genomic and transcriptomic landscapes. Nat Commun. 2016;7:11479.

10. Ritchie ME, Phipson B, Wu D, et al. limma powers differential expression analyses for RNA-sequencing and microarray studies. Nucleic Acids Res. 2015;43:e47.

11. Jezequel P, Campone M, Gouraud W, et al. bc-GenExMiner: an easy-to-use online platform for gene prognostic analyses in breast cancer. Breast Cancer Res Treat. 2012;131:765-775.

12. Rakha EA, Pinder SE, Bartlett JM, et al. Updated UK recommendations for HER2 assessment in breast cancer. J Clin Pathol. 2015;68:93-99.

13. Rakha EA, Agarwal D, Green AR, et al. Prognostic stratification of oestrogen receptor-positive HER2-negative lymph nodenegative class of breast cancer. Histopathology. 2017;70:622-631. 
14. Hammond ME, Hayes DF, Dowsett M, et al. American Society of Clinical Oncology/College of American Pathologists guideline recommendations for immunohistochemical testing of estrogen and progesterone receptors in breast cancer (unabridged version). Arch Pathol Lab Med. 2010;134:e48-e72.

15. McCarty KS Jr, Miller LS, Cox EB, Konrath J, McCarty KS,Sr. Estrogen receptor analyses. Correlation of biochemical and immunohistochemical methods using monoclonal antireceptor antibodies. Arch Pathol Lab Med. 1985;109:716-721.

16. Kalikin LM, Bugeaud EM, Palmbos PL, Lyons RH Jr, Petty EM. Genomic characterization of human SEC14L1 splice variants within a $17 \mathrm{q} 25$ candidate tumor suppressor gene region and identification of an unrelated embedded expressed sequence tag. Mammalian genome: official journal of the International Mammalian Genome. Society. 2001;12:925-929.

17. Sinclair CS, Rowley M, Naderi A, Couch FJ. The 17q23 amplicon and breast cancer. Breast Cancer Res Treat. 2003;78:313-322.

18. Agell L, Hernandez S, Nonell L, et al. A 12-gene expression signature is associated with aggressive histological in prostate cancer: SEC14L1 and TCEB1 genes are potential markers of progression. Am J Pathol. 2012;181:1585-1594.

19. Burdelski C, Barreau Y, Simon R, et al. Saccharomyces cerevisiae-like 1 overexpression is frequent in prostate cancer and has markedly different effects in Ets-related gene fusion-positive and fusion-negative cancers. Hum Pathol. 2015;46:514-523.

20. Zhao S, Xu C, Qian $\mathrm{H}$, et al. Cellular retinaldehyde-binding protein-like (CRALBPL), a novel human Sec14p-like gene that is upregulated in human hepatocellular carcinomas, may be used as a marker for human hepatocellular carcinomas. DNA Cell Biol. 2008;27:159-163.

21. Carver BS, Tran J, Gopalan A, et al. Aberrant ERG expression cooperates with loss of PTEN to promote cancer progression in the prostate. Nat Genet. 2009;41:619.
22. Puc J, Keniry M, Li HS, et al. Lack of PTEN sequesters CHK1 and initiates genetic instability. Cancer Cell. 2005;7:193-204.

23. Stern HM, Gardner H, Burzykowski T, et al. PTEN loss is associated with worse outcome in HER2-amplified breast cancer patients but is not associated with trastuzumab resistance. Clin Cancer Res. 2015;21:2065-2074.

24. Yu J, Yu J, Mani RS, et al. An integrated network of androgen receptor, polycomb, and TMPRSS2-ERG gene fusions in prostate cancer progression. Cancer Cell. 2010;17:443-454.

25. Shah AV, Birdsey GM, Randi AM. Regulation of endothelial homeostasis, vascular development and angiogenesis by the transcription factor ERG. Vasc Pharmacol. 2016;86:3-13.

26. Polson ES, Lewis JL, Celik H, et al. Monoallelic expression of TMPRSS2/ERG in prostate cancer stem cells. Nat Commun. 2013;4:1623.

27. Banin-Hirata BK, de Oliveira CEC, Losi-Guembarovski R, et al. The prognostic value of regulatory $\mathrm{T}$ cells infiltration in HER2enriched breast cancer microenvironment. Int Rev Immunol. 2018;37:144-150.

28. Mohammed RA, Ellis IO, Lee AH, Martin SG. Vascular invasion in breast cancer; an overview of recent prognostic developments and molecular pathophysiological mechanisms. Histopathology. 2009;55:1-9.

29. Mousley CJ, Tyeryar KR, Vincent-Pope P, Bankaitis VA. The Sec14-superfamily and the regulatory interface between phospholipid metabolism and membrane trafficking. Biochim Biophys Acta. 2007; 1771:727-736.

30. Ribeiro FM, Ferreira LT, Marion S, et al. SEC14-like protein 1 interacts with cholinergic transporters. Neurochem Int. 2007;50:356-364.

31. Li M-T, Di W, Xu H, et al. Negative regulation of RIG-I-mediated innate antiviral signaling by SEC14L1. J Virol. 2013; 87:10037-10046. 